\title{
Differences in injury distribution in professional and recreational snowboarding
}

This article was published in the following Dove Press journal:

Open Access Journal of Sports Medicine

16 April 2015

Number of times this article has been viewed

\section{Christian Ehrnthaller ${ }^{1}$ \\ Heinz Kusche ${ }^{2}$ \\ Florian Gebhard'}

'Department of Traumatology, Hand-, Plastic-, and Reconstructive Surgery, Center of Surgery, University of Ulm, Germany; ${ }^{2}$ Department of Traumatology and Orthopedics, Garmisch-Partenkirchen Medical Center, Germany
Correspondence: Christian Ehrnthaller Department of Traumatology, Hand-, Plastic-, and Reconstructive Surgery, University of Ulm, Albert-Einstein Allee 23, 8908I Ulm, Germany $\mathrm{Tel}+497315005455$ I

Fax +49 73I 50054502

Email christian.ehrnthaller@uniklinik-ulm.de
Introduction: Little is known about the distribution of injuries in competitive snowboarding and there are no reports describing long-term complications of professional snowboarding. To examine the injury distribution and long-term impairments in active and retired professional snowboarders, a retrospective data analysis was performed using a questionnaire. As a control group, injuries of recreational snowboarders of a southern German university clinic were examined.

Methods: Retired and active snowboarders were identified from the result lists of the International Ski Federation (FIS) and from the current team lists of the German-speaking European national snowboard teams. After identification, a questionnaire regarding injury distribution/ treatment was sent to the athletes and the filled-in questionnaires were subsequently evaluated. Injuries in recreational snowboarding were evaluated by analyzing the medical records and surgical reports for a seven-year period.

Results: Compared to the available literature, significantly more severe injuries that necessitated surgical treatment in recreational snowboarders were documented. Most injuries accounted for wrist fractures but also many nonunions and spinal fractures were recorded. Between active professionals, distinct differences in injury distribution were found. The number of days off differed greatly with less days in speed disciplines (35.5 days) and the maximum number of days off in snowboardcross (51 days). Injury distribution varied clearly with more injuries of the upper extremity and ankle in speed disciplines compared to snowboardcross and freestyle professionals, who exhibited more injuries to the lower extremity and head. Freestyle athletes used significantly less protectors compared to speed $(P=0.01)$ and snowboardcross athletes $(P=0.00)$. An analysis of retired professionals revealed a higher number of impairments in daily life and a significantly higher number of days off snowboarding $(P=0.018)$.

Conclusion: Knowledge about snowboard-specific injuries is a requirement for an appropriate treatment of these patients. This first description of long-term impairments after professional snowboarding highlights the importance of the development of specific strategies to prevent daily life impairments after a professional sports career.

Keywords: snowboarding, injury-distribution, professional snowboarding

\section{Introduction}

Snowboarding is a relatively young sport that has been developed in the mid 1960s by binding two skis together and using it to surf on snow. Continuous development of ski technology and the introduction of steel edges and bindings made the sport more fun and began to attract mainly the young generation in the 1970 s and $1980 \mathrm{~s} .{ }^{1}$ The first contests were held in the late 1970s, while the first national championships were held in 1982 followed by the first World Cup in $1991 .^{2}$ With the increased number of people 
attracted to the sport and the development from a trend to a mass sport in the 1990s, snowboarding became an Olympic sport when the disciplines halfpipe and giant slalom were included in the Winter Olympics in Nagano in 1998. ${ }^{2,3}$ In the 2014 Sochi Olympics, snowboarding was represented by five disciplines, with the traditional disciplines halfpipe, parallel giant slalom, and snowboardcross, and also with a premiere of parallel slalom and slopestyle. ${ }^{1}$

In 2010, there were an estimated 11.5 million people skiing and 8.2 million snowboarding in the United States of America. ${ }^{3}$ In Germany at the moment, there are about 2.2 million active snowboarders. ${ }^{4}$

According to a major Swiss insurance company, snowboarding represents the most dangerous sport after soccer and ice hockey. Overall, the risk of getting injured was estimated to be about twice as high as for skiing as snowboarders represented almost half of the patients in the emergency departments but only a quarter of all participants on the slope. ${ }^{5,6}$ Recent studies were able to document an almost equal incidence of injuries between snowboarding and skiing probably due to the increasing incidence of freesking injuries. ${ }^{7}$ Approximately $40 \%-59 \%$ of all injuries occur in beginner snowboarders ${ }^{6,8}$ with $13 \%-23 \%$ of injuries occurring on the first day of snowboarding. ${ }^{5,9}$ The typical injured snowboarder is male and between the age of 15-29. Several studies have been performed to present the different injury patterns in snowboarding compared to skiing. ${ }^{10}$ As early as 1985, Shealy and Sundman showed the differences between snowboarding and skiing, with a higher rate of wrist and ankle injuries in the latter sport. ${ }^{11}$ In 2010, a case-control study was published analyzing snowboard injuries compared to skiing injuries in persons over 18 years. In the group of injured snowboarders, injuries of the wrist, ankle, and shoulder were more frequent, while most of the injuries in skiers were seen in the knee joint. ${ }^{3}$ Over a period of 18 years, an alteration in the injury pattern of snowboarding was demonstrated. The number of sprains of the medial collateral knee ligament and ankle injuries decreased, and the fractures of the clavicle increased. ${ }^{3}$ The most frequent type of injury in snowboarding was a wrist injury; for skiers, it was a sprain of the anterior cruciate ligament (ACL).

Fewer studies investigated the incidence of injuries in professional snowboarding. Torjussen and Bahr were able to show a different injury pattern for competitive snowboarders with fewer wrist injuries but more knee and back injuries. Within the group of competitive snowboarders, World Cup competitors had fewer injuries compared to national level competitors. ${ }^{2}$ Another study was able to demonstrate an increasing injury severity for an elevated skill level with a higher number of multiple injuries and the trauma of the trunk. ${ }^{12}$ The type of discipline in snowboarding has a high impact on the risk of injury and the expected injury distribution. The injury incidence is relatively low for disciplines such as parallel slalom and parallel giant slalom while athletes in disciplines with jumps and body contact such as snowboardcross, halfpipe, and slopestyle are more likely to get injured. ${ }^{2}$ The increasing readiness to assume a risk was demonstrated in a study comparing qualification and final runs in a snowboardcross event revealing a higher injury incidence of final runs. ${ }^{13}$

With the main focus on acute injuries during a competitive season to describe discipline-specific differences, the aforementioned studies were not able to answer the question regarding long-term impairments in professional snowboarding. Furthermore, there is no study evaluating the injuries and impairments in retired professional snowboarders.

Therefore, the main goal of this study was to describe the possible long-term impairments in active and retired professional snowboarders by analyzing all suffered injuries of each athlete during their professional career. With this additional information, the importance of developing injuryand discipline-specific prevention strategies can be estimated, without which it would be hard to gain the attention and ensure the understanding of the athletes and trainers.

\section{Methods}

The study was approved by the ethical committee of the University of Ulm and was conducted in accordance with the international standards, ethical guidelines, and the Declaration of Helsinki.

\section{Recreational snowboarders}

To assess the injury pattern for recreational snowboarders, an evaluation of all patients (all ages) with snowboard injuries who presented to the Department for Traumatology, Hand-, Plastic-, and Reconstructive Surgery of the University of Ulm from January 1, 2005 until December 31, 2012 was carried out. The university hospital of Ulm is a hospital providing maximum care with specialized departments and is located near the Alps, representing the second largest maximum care hospital in one of the most popular areas for winter sports in Germany.

The identification of the patients was carried out by a free text search in our clinical electronic database. Search terms were "snowboard", "snowboard-injury", "snowboarding", and "boarding". Surgical protocols and medical reports were 
analyzed. The documents were evaluated for age, sex, type of injury, treatment (operative/conservative), complications, and reoperations. The evaluation of recreational snowboarders included 186 patients with a total of 218 injuries.

\section{Active professional snowboarders}

The athletes were identified according to the national snowboard team lists of the European countries with German as a community language (Germany, Austria, and Switzerland). Athletes from all disciplines (speed disciplines, freestyle, snowboardcross) and from cadre A-C/D were included.

After the approval of the study by the local ethical committee, an anonymous questionnaire and patient information with informed consent was generated and sent to the athletes by post or electronically. After completion, the anonymous questionnaires were received by the investigator either by post or electronically. Ninety eight injuries (only single, no multiple injuries) in 38 individual athletes in three different disciplines were documented with an overall response rate of $71 \%$. From the shipment to the evaluation of the questionnaires, it took 24 months.

The questionnaire included information on the age, sex, experience (snowboard overall, professional experience), types of injuries, action while the injury took place (contest, training, freeride), protectors, type of boots (soft/hard), treatment (operative/conservative), days off, impairment in daily life, return to sports, and ability to perform snowboarding at the same level as that before injury. All collected data were analyzed in detail and by comparison of arithmetic means. Injuries were analyzed overall and specifically for each discipline.

\section{Retired professional snowboarders}

The group of retired professional snowboarders was identified according to former national team lists and World Cup results of all disciplines (parallel slalom and giant slalom, snowboardcross and freestyle) from 1998-2002. All former athletes from cadre A-C/D whose names were listed in these documents were identified as possible candidates. All athletes whose postal or email address could be identified were included in the study. Next, the athletes were contacted, and the questionnaire together with forms for informed consent and patient information was sent to them by post or electronically. The questionnaire used in this group was identical to the one sent to active professional snowboarders with additional questions regarding the time since retirement and the reason for retirement from the professional career. In this group, 57 injuries in 19 individuals from all disciplines (parallel slalom and giant slalom, snowboardcross and freestyle) were documented and combined. The overall response rate was $17 \%$. From the shipment to the evaluation of the questionnaires, it took 24 months.

\section{Statistics}

All collected data were analyzed in depth and by comparison of arithmetic means. Injuries were analyzed overall and specifically for each discipline. For statistical evaluation, a nonparametric Mann-Whitney test and the Chi-square test (IBM SPSS Statistics 19.0; SPSS Inc., IBM, Armonk, New York, USA) were conducted. Results with $P \leq 0.05$ were considered statistically significant.

\section{Results}

\section{Recreational snowboarders}

The mean overall age was 23.3 years with 23.2 for male and 23.6 for female snowboarders. There were nearly twice as many male participants (126) compared to female participants (60).

In the cohort of recreational snowboarders, 125 injuries required surgical treatment while 93 injuries could be treated conservatively. Of all the patients, 21 had multiple injuries and 23 had complications that were subsequently treated. The most frequent complication was nonunion in eleven cases, followed by five dislocations and five early metal removals due to prolonged pain at the implantation site. Most of the nonunion complications were seen in fractures of the scaphoid with seven of them treated with an iliac crest autograft. In all of those cases $(n=7)$, the reason for nonunion was because the patients did not see a doctor after injury and therefore no specific treatment was initiated.

A global look at the injury distribution with respect to the different body areas demonstrated a predominant involvement of the upper extremity with $72 \%$, followed by injuries of the spine and lower extremity with $9 \%$. The lowest rate of injuries was seen in the head with 5\% (Figure 1).

The most frequent injuries were injuries of the wrist and hand with a total number of 77 injuries. Within this group, the majority of the injuries were distal radius fractures $(n=45)$ followed by fractures of the scaphoid $(n=16)$ and other fractures of the hand $(n=16)$.

Most injuries were in the upper extremity, with 12 clavicular fractures, ten elbow injuries, and nine acromioclavicular (AC)-joint sprains. There was a relatively large number of spine fractures documented $(n=13)$. There were eight head injuries in total and seven ankle injuries (Figure 2). There 


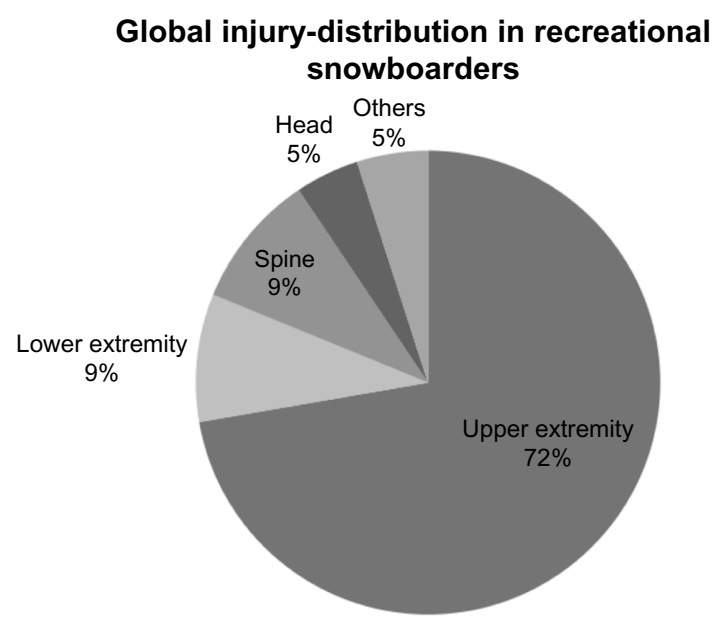

Figure I Global injury-distribution in recreational snowboarders in \% of all evaluated injuries at the university clinic of Ulm.

were only two documented knee injuries that were both ruptured menisci.

\section{Active professional snowboarders}

The mean overall age of active professional snowboarders was 22.2 years, with $58 \%$ male athletes. Overall, one third of all injuries were treated operatively. All athletes stated a full recovery after their injuries with the same level of success in competitions as that before the injury, but 13 athletes reported impairments in daily life. Most of the injuries happened during training $(\mathrm{n}=48)$ and during competition $(\mathrm{n}=33)$, and only a few $(n=7)$ accidents occurred while freeriding (not specified for eleven injuries). Although all athletes are competitive, professional riders, only $74 \%$ of the athletes wore protection gear at the time of injury. The average days off professional sports activity due to the injury was 43.0 days (Table 1 ). The global injury-distribution was dominated by injuries of the upper extremity at $51 \%$, followed by that of the lower extremity at $26 \%$, and others (22\%) (Figure $3 \mathrm{~A}$ ). The most frequent injuries for all disciplines were head concussions and fractures to the distal radius (Figure 4).

With respect to the different snowboarding disciplines, remarkable differences regarding injury distribution were found. The largest group represented the athletes of the speed disciplines (parallel slalom and parallel giant slalom). Here, injuries to the hand and upper extremity were predominant. Sixty percent of all injuries accounted for the upper extremity and in this case, injuries of the hand represented the largest group (37\%). In contrast to the other disciplines, a large number of ankle injuries were documented (12.5\%) (Figure 3B).

Another striking difference was documented regarding the days off professional snowboarding after injury. Here, the athletes from the speed disciplines had the least number of 35.5 days. In this group of athletes, two times more injuries occurred while training than during competitions (Table 1).

The most frequent injury was a fracture of the wrist followed by fractures of the fingers (Figure 3B). A closer look at ankle injuries demonstrated a dominance of collateral ligament ruptures and fractures.

A great variance was visible in the use of protection gear. The freestyle athletes used significantly less protection gear compared to their colleagues from the speed disciplines ( $P=0.01)$ and the snowboardcross discipline $(P=0.00)$.

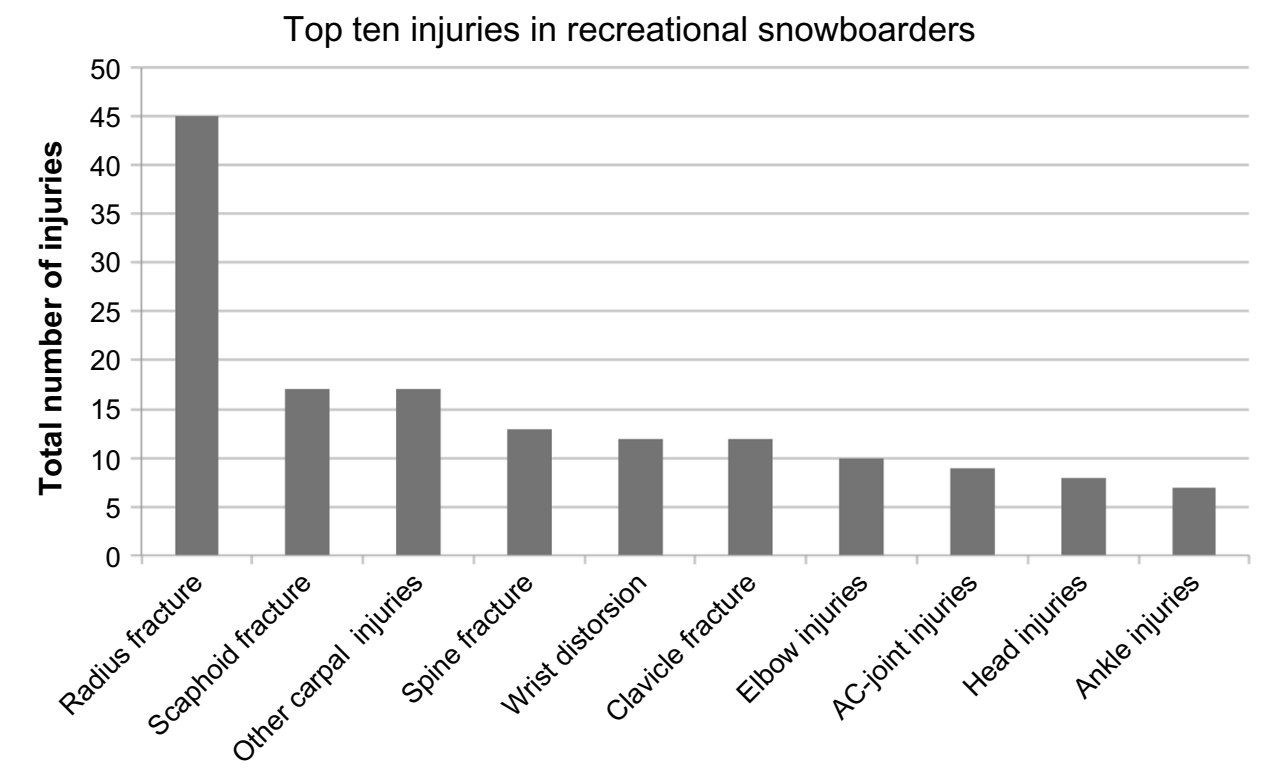

Figure 2 Most frequent injuries, of all evaluated snowboard injuries at the university clinic of Ulm, in total numbers. Abbreviation: AC, acromioclavicular. 
Table I Descriptive statistics of German-speaking national snowboard teams.

\begin{tabular}{|c|c|c|c|c|c|}
\hline Characteristics & $\begin{array}{l}\text { Race ( } n=21 ; \\
44 \text { injuries) }\end{array}$ & $\begin{array}{l}\text { Freestyle }(n=8 ; \\
25 \text { injuries) }\end{array}$ & $\begin{array}{l}\text { Snowboardcross } \\
\text { ( } n=9 ; 30 \text { injuries) }\end{array}$ & $\begin{array}{l}\text { Active professionals } \\
\text { ( } n=39 ; 99 \text { injuries) }\end{array}$ & $\begin{array}{l}\text { Retired professionals } \\
\text { ( } n=19 ; 57 \text { injuries) }\end{array}$ \\
\hline Mean age (in years) & 25.6 & 18.6 & 24.0 & 22.2 & 34.5 \\
\hline Sex (male/female) (total numbers) & $12 / 9$ & $5 / 3$ & $5 / 4$ & $22 / 16$ & $14 / 5$ \\
\hline Snowboarding experience (in years) & 16.7 & 10.1 & 16.1 & 13.6 & 20.7 \\
\hline Professional experience (in years) & 8.5 & 4.8 & 7 & 6.4 & 7.5 \\
\hline Boots (soft/hard) (total numbers) & $4 / 40$ & $25 / 0$ & $30 / 0$ & $59 / 40$ & $19 / 38$ \\
\hline Protectors (in \%) & $70 \% *$ & $36 \% *$ & $93 \%$ & $74 \%$ & $37 \% *$ \\
\hline $\begin{array}{l}\text { Activity when injured (contest/training/ } \\
\text { freeride/ns) (total numbers) }\end{array}$ & $12 / 24 / 2 / 6$ & $8 / 13 / 4 / 0$ & $13 / 1|/| / 5$ & $33 / 48 / 7 / 11$ & $16 / 30 / 2 / 9$ \\
\hline $\begin{array}{l}\text { Treatment (operative/conservative) } \\
\text { (total numbers) }\end{array}$ & $14 / 30$ & $8 / 17$ & $12 / 18$ & $34 / 65$ & $17 / 39$ \\
\hline Days off (in days) & 35.5 & 46.7 & 51.0 & 43.0 & $79.1 *$ \\
\hline $\begin{array}{l}\text { Equal level of success after injury } \\
\text { (total numbers) }\end{array}$ & $21 / 21$ & $8 / 8$ & $10 / 10$ & $39 / 39$ & $13 / 19 *$ \\
\hline Return to sports (total numbers) & $21 / 21$ & $8 / 8$ & $10 / 10$ & $22 / 22$ & $17 / 19$ \\
\hline Daily life impairment (total numbers) & $7 / 21$ & $2 / 8$ & $4 / 5$ & $13 / 34$ & $10 / 19$ \\
\hline Year since retirement (in years) & NA & NA & NA & NA & 8 \\
\hline Retirement due to injury (total numbers) & NA & NA & NA & NA & $5 / 19$ \\
\hline
\end{tabular}

Notes: NA, not applicable; $* P \leq 0.05$.

The riders of the freestyle discipline showed predominantly injuries of the upper extremity and knee. We were able to document $22 \%$ of wrist fractures and $11 \%$ fractures of the clavicle. Another large group in this discipline was ACL-rupture, accounting for $15 \%$. Twenty six percent of all injuries occurred in other body parts (Figure 3C).

A Global injury distribution in active professional

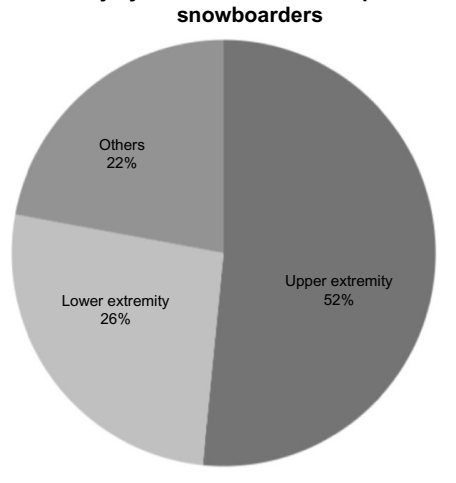

C Top injuries, national freestyle teams

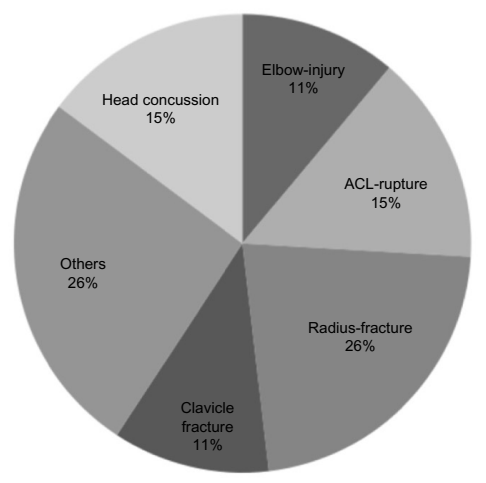

Although the average number of days off was almost as high as that in the group of snowboardcross athletes (46.7), remarkably only $36 \%$ of the athletes wore protective gear at the time of injury. All athletes stated full recovery with the same level of successful riding after injury and no impairments in their daily life. Most of the injuries occurred during

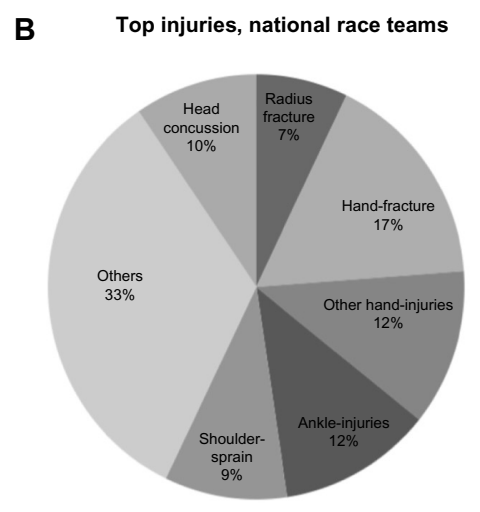

D Top injuries, national snowboardcross teams

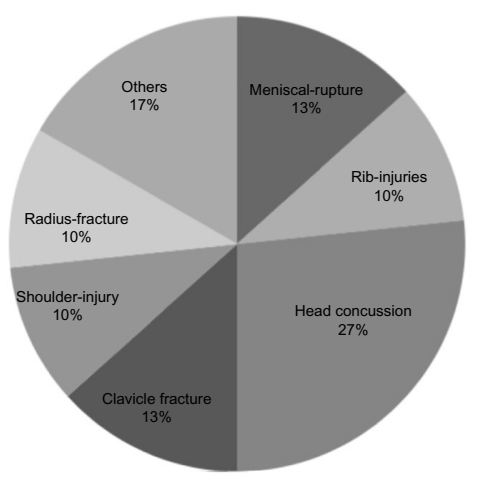

Figure 3 Diagrams of the injury distribution of the 2012/2013 German-speaking national snowboard teams in percent.

Notes: (A) global injury-distribution of all disciplines; (B-D) predominant injuries regarding the different snowboarding disciplines, in percent. Abbreviation: $A C L$, anterior cruciate ligament. 


\section{Top ten injuries in active professional snowboarders}

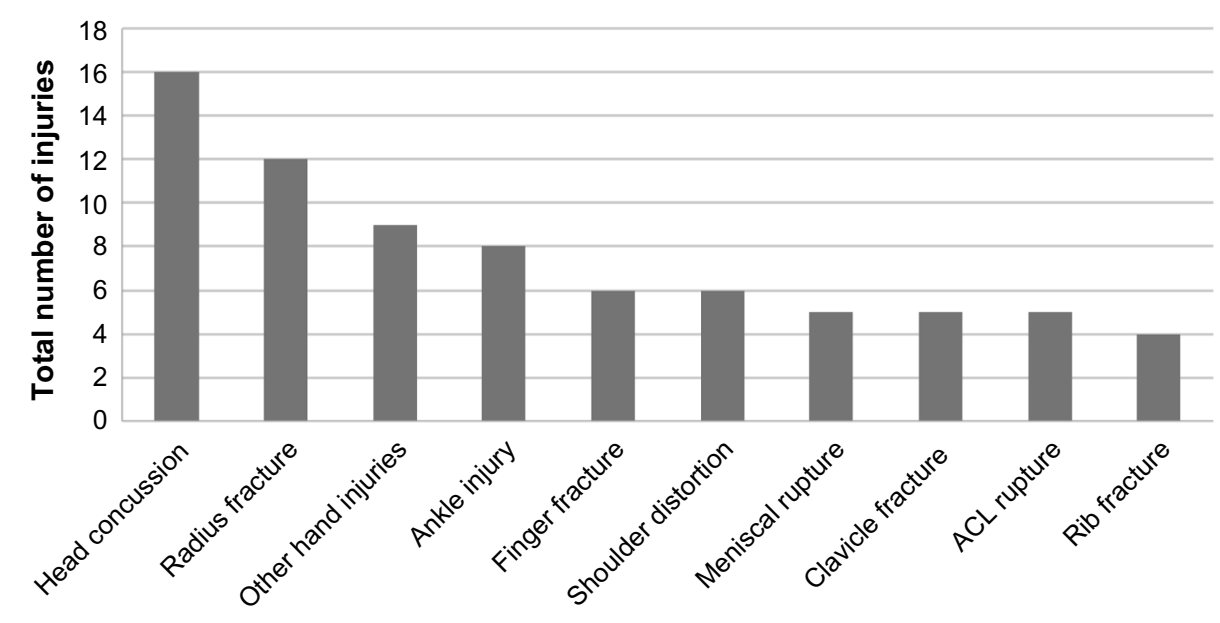

Figure 4 Most frequent injuries of all documented snowboard injuries for the 2013/2014 German-speaking national snowboard teams, in total numbers. Abbreviation: $\mathrm{ACL}$, anterior cruciate ligament.

training $(52 \%)$ followed by injuries during competitions $(32 \%)$ (Table 1).

The group of athletes riding snowboardcross represented a very heterogeneous group with the most variety of injuries. Most injuries occurred in the head at 27\% followed by shoulder injuries at $23 \%$. But also many injuries of the thorax $(10 \%)$ and knee with ruptures of the menisci (13\%) were apparent (Figure 3D). The type of riding during injury was almost equally distributed between contest and training. In contrast to the other groups, almost half of the athletes commented about impairment in their daily life, but all athletes stated that they achieved the same level of success after injury. In the group of snowboardcross riders, the highest number of days off professional sports was seen with 51.0 days average. Most of the riders wore protective gear $(93 \%)$ (Table 1).

\section{Retired professional snowboarders}

The mean age of athletes in this group was 34.5 years with 20.7 years of snowboarding experience. The average professional snowboarding experience was 7.5 years with a mean time since retirement from professional snowboarding of 8 years.

In contrast to active professional snowboarders, significantly more $(32 \%)$ athletes stated inability to snowboard at the same level as that before injury $(P=0.001)$. Twenty six percent had to abandon their professional career due to injury reasons with 52\% reporting about impairments in their daily life; two athletes even reported that they are not able to snowboard anymore.
Another striking difference observed in the study is the number of days off after injury. In the group of retired professional snowboarders, we observed 79.1 days off compared to 43.0 days in the group of active professional snowboarders $(P=0.018)$. Active professional snowboarders nowadays wear protective gear in $74 \%$ of all cases (Table 1 ), whereas retired athletes only stated to have worn protective gear in $37 \%$ of all accidents, a result that reached significance $(P=0.001)$. In the group of retired professional snowboarders, twice as many injuries happened during training rather than competition as in the group of active professionals (Table 1).

Looking at the injury distribution, we find that predominantly the upper extremity was affected with $55 \%$, followed by $35 \%$ of the lower extremity. The main affected joints were the wrist (41\%) and shoulder $(23 \%)$ followed by the knee $(21 \%)$ and ankle with $15 \%$ of all injuries (Figure $5 \mathrm{~A}$ and $\mathrm{B})$.

Contrary to the groups of recreational and active professional snowboarders, fractures of the wrist were rarely seen. The most frequent injuries were injuries of the finger followed by clavicle fractures and metacarpophalangeal ulnar collateral ligament (MCP-UCL) injuries of the thumb. This was followed by knee and ankle injuries (Figure 6).

\section{Discussion}

This study represents a first attempt to describe the different injury patterns observed in the case of recreational snowboarders and professional competitive athletes. 

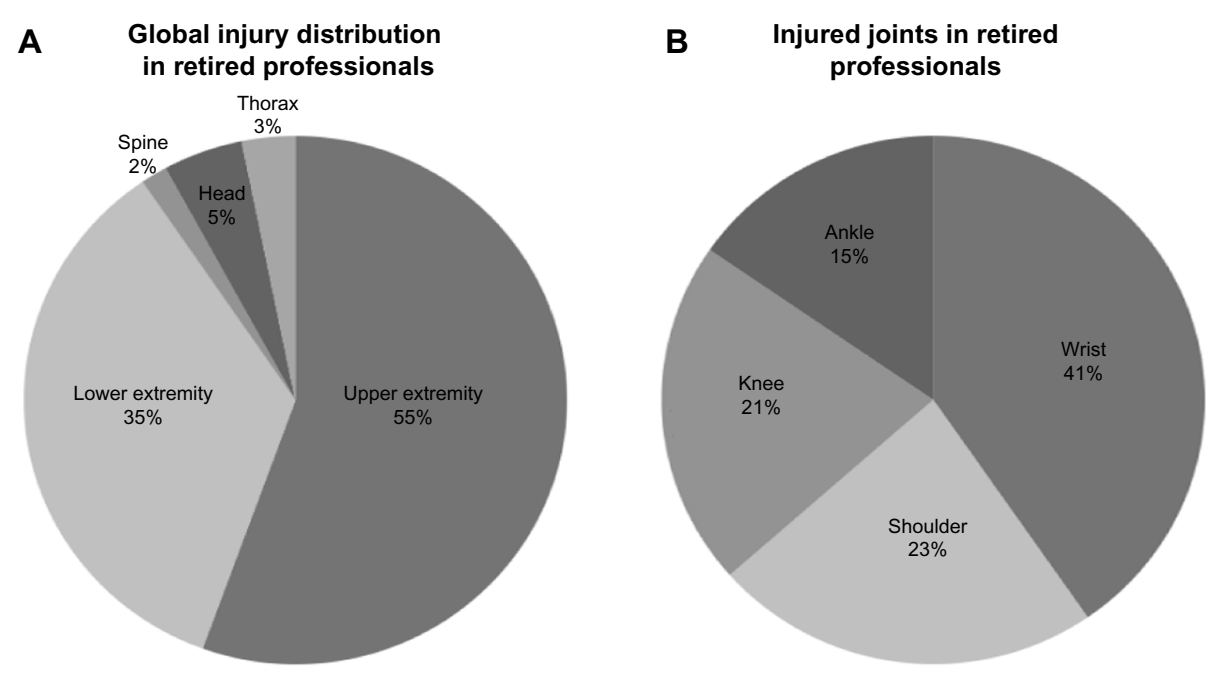

Figure 5 Diagrams of the injury distribution in retired snowboard professionals in percent.

Notes: (A) global injury-distribution regarding body area; (B) predominantly injured joints, in percent.

In addition, this study is the first to describe long-term health impairments in a collective cohort of retired professional snowboarders.

\section{Recreational snowboarders}

Relating to age and sex, the results in our collective cohort are in agreement with the literature, indicating the typical snowboarder was injured at an age between 19 and 23 and a proportion between male and female of $2: 1.14,15$

In this study collective, the injuries of the upper extremity accounted for $72 \%$ of all injuries. Comparing with the available literature, this rate is two times the normal. The rate of injuries to the upper extremity varies from $30 \%$ to $60 \% 0^{5,8}$ in studies conducted before the year 2000; in the latest literature, the prevalence is found to be around $40 \%-60 \%{ }^{3,16,17}$

A possible reason for this overrepresentation of upper extremity injuries may be due to the presence of a department for hand surgery in our hospital. This is represented in the large number of scaphoid fractures and other carpal fractures, which in total account for $16 \%$ of all injuries. Other studies show a prevalence of scaphoid fractures between $2 \%$ and $3 \%$. ${ }^{16,18}$

Taking a closer look at the scaphoid fractures raises another interesting point. In 17 scaphoid fractures, seven developed a nonunion, with all patients requiring an iliac crest autograft. Interestingly, in five of these patients, the injury was not detected by a previous doctor. This clearly

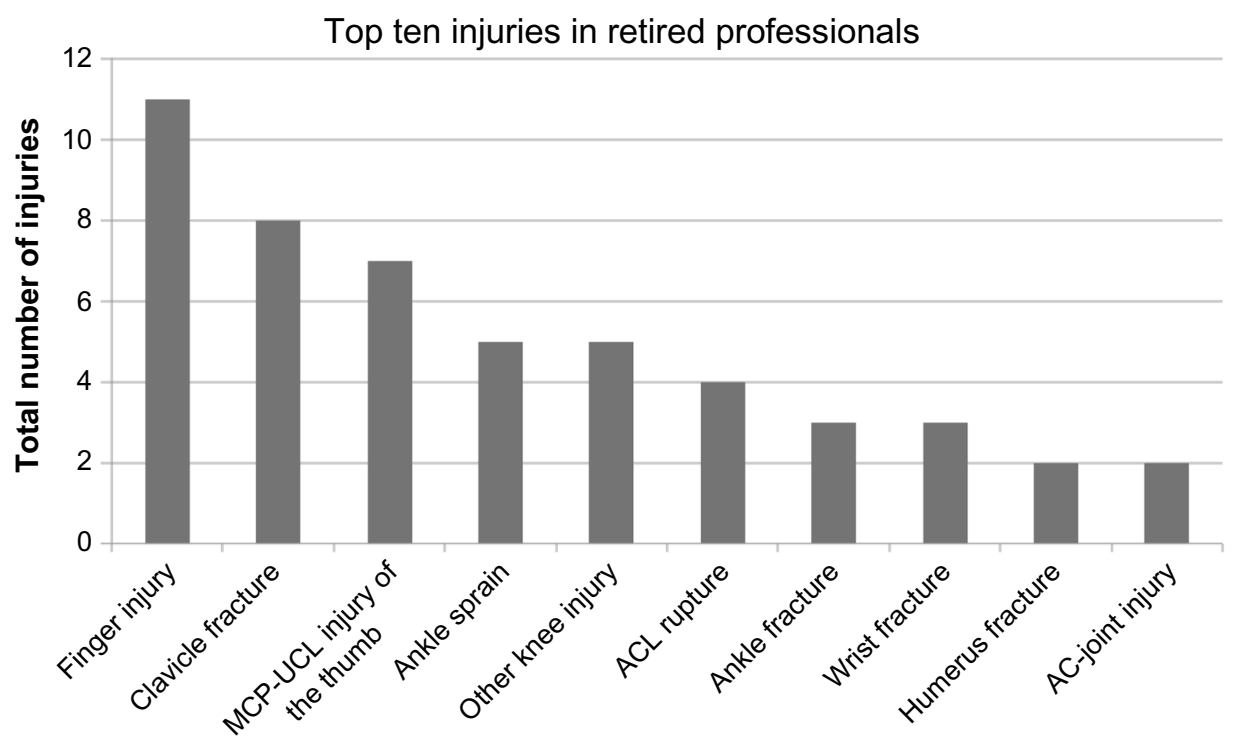

Figure 6 Most frequent injuries of all documented snowboard injuries for retired professional snowboarders, in total numbers. Abbreviations: $A C L$, anterior cruciate ligament; MCP-UCL, metacarpo-phalangeal ulnar collateral ligament; AC, acromioclavicular. 
demonstrates that the scaphoid fracture is an injury that can be overseen easily and should be a focus of diagnostic procedures in patients with prolonged pain after a fall onto the wrist.

While injuries to the upper extremity were overrepresented, lower extremity injuries were relatively underrepresented in our collective. However, the literature shows a large variety based on the age of the study. While in newer studies the rate of lower extremity injuries is around $12 \%-14 \%, 3,12$ this number varies in older studies with values from $38 \%-59 \%$. $^{6,11}$

The reason for the reduced number in our collective may be seen in the prescreening of the patients directly at medical centers near the ski resort or the general practitioner near home. As most of the lower extremity injuries are bruises and sprains, ${ }^{15,19}$ it is likely that patients suffering from these injuries are not admitted to the nearest university hospital.

The third largest collective in our study represents fractures to the spine, at $9 \%$ of all injuries. This was significantly higher than the $3 \%$ that was previously reported in the literature. ${ }^{20}$ This may be an effect of more severe injuries generally seen in the patient collective of university hospitals in Germany. This is underlined by the fact that $11 \%$ of all treated patients had multiple injuries.

Another difference of our collective compared to the literature is the ratio between operative and conservative treatment. While normally $3 \%-10 \%{ }^{14}$ of injuries are treated surgically, in our collective $67 \%$ of all injuries were treated with a surgical procedure. A possible reason for this deviation may be the aforementioned prescreening of injuries through the medical centers and general practitioners near the ski resorts, filtering most of the injuries with minor injury severity not requiring surgical treatment. As these minor injuries represent $64 \%$ of all injuries ${ }^{14}$ this effect seems to be the main reason.

Generally, our results should not be seen as the "true" collective of snowboard injuries, but may represent a deeper look into the second line of health care represented by a large university clinic with a spectrum of snowboard injuries that demanded further assistance by specialized departments, and in most of the cases, surgical treatment.

\section{Active professional snowboarders}

Combining all sub-disciplines in active professional snowboarding to one large group, the results according to age, sex, and global injury-distribution regarding the different body parts correlate well with the available statistical data from the literature.
It is generally known that with increasing skill levels of recreational athletes, the injury severity increases accordingly. ${ }^{12,21}$ Subdividing the professional athletes, Torjussen and Bahr were able to demonstrate a lower level of injury severity at the World Cup level compared to the national competition level. ${ }^{2}$ This higher rate of injury severity in professional athletes is displayed in the higher number of operatively treated injuries in our cohort.

While a large number of athletes stated impairment in their daily life, not a single athlete stated to be less successful than before injury. This result should be interpreted with caution, as all athletes were still actively competing and may understate the fact of impairment for their professional riding.

Although for both active professional and recreational snowboarders, the most common injury was a fracture to the wrist, professional snowboarders exhibited a less pronounced injury pattern of the wrist and hand with more injuries to the knee joint. This can be explained with the fact that the most common injury mechanism of wrist fractures in snowboarding represents a typical fall of the novice or an inexperienced snowboarder with the edge catching snow causing a fall onto the outstretched hands. ${ }^{14,17}$ The highest prevalence of such injuries is found on the first day of snowboarding. ${ }^{14}$ While the mechanism of injury resembles that of novices, the reason for falling of a professional snowboarder is completely different. Snowboard beginners typically lack edge-control and therefore they fall and injure themselves at the outstretched hands. In competitive snowboarding, the reason for a fall on the hand is not a lack of edge-control but a mistake while performing a jump or a collision with the gate or another snowboarder in the snowboardcross-discipline.

A more distinct view to the injury distribution can be found when looking at the different disciplines of professional snowboarding. In the group of speed disciplines (parallel slalom and giant slalom), the lowest rate of days off was found. This is in accordance with a study by Torjussen and Bahr according to which the speed disciplines have the smallest injury severity among all snowboarding disciplines. ${ }^{2,22}$ Another major difference of speed- compared to other snowboard disciplines was demonstrated regarding the injury distribution with predominance of injuries to the hand and fingers followed by injuries to the ankle.

These results are in accordance with other studies that state about $50 \%$ of all injuries in alpine professional snowboarding occur in the fingers. ${ }^{2}$ The reason for these types of injuries is uniformly reported to be the impact of the finger onto the pole or the gate panels. 
Contrary to this, the reason for the relatively high number of injuries to the ankle joint remains unclear. Kirkpatrick et al reported that about $73 \%$ of all ankle injuries are caused when wearing soft boots, but this cannot be affirmed in our cohort. ${ }^{23}$ One would believe that the rigid nature of hard boots would not give enough range of motion to create this kind of fracture, but obviously there is an unknown mechanism creating enough force even when snowboarders wear rigid snowboard hard boots. One possible explanation may be found in the development of the snowboard equipment since the study of Kirkpatrick et al. At that time, soft boots were very soft creating a very high range of motion; additionally, the available bindings did not give much support either. Therefore, while falling, very excessive over extensions to the ankle joint were possible in the past, resulting in these kinds of injuries. The design and behavior of soft boots nowadays have completely changed. During the last century, the industry developed soft boot equipment and bindings that are almost as stiff as the hard boots used in the mid 1990s. Therefore, the maximal movements described earlier are not possible anymore, possibly rescuing the athlete from injuries to the ankle. The hard boot equipment also evolved and development made the equipment harder and less flexible. Besides stronger and less flexible hard boots, the binding technique progressed, creating a stronger connection between boots and the snowboard. Formerly, while falling with hard boots, a bending movement between the binding and boots was possible; this reduced the forces on the ankle and foot. Nowadays, this movement is largely eliminated due to the boots fixed rigidly in the binding with almost no possible bending of the boots in the binding. This results in a hindered movement of the foot remaining basically in its position, and a compensatory over-movement of the ankle in the more flexible boot, compared to the sole/boot-binding interface, thus resulting in ankle injuries. The clarification of the exact mechanism and consecutive development of prevention strategies and alternative binding/boot designs should be the target of future studies.

In the group of freestyle athletes (halfpipe, slopestyle, and big air) injuries to the wrist and knee joint were predominant. This is in accordance with the literature, with the reason of injury mostly being a fall during a jump. Within freestyle snowboarding, there are two major reasons for falls. First of all, there is the possibility of a snowboarder to land a jump not in the transition area of the halfpipe or the big air jump but in the flat area. This typically results in a strong load on the knee capable of creating shear forces and a bending momentum powerful enough to destroy knee ligaments. The other mechanism is a classical fall backwards when the edge catches snow, or a fall onto the head and the upper extremity while doing upside-down flips. In the latter cases, the athlete tries to prevent the impact using his hands with consecutive injuries to the upper extremity.

Surprisingly, the amount of protective gear used was significantly lower in the freestyle athletes. As it is generally known that protective gear is capable of reducing the number of wrist injuries, ${ }^{18}$ wearing protectors should be a promising strategy in the case of these athletes as well. ${ }^{1}$

The maximum variability in injuries was seen in the group of snowboardcross athletes.

Most injuries occurred in the head (27\%) and shoulder (23\%), but several injuries also occurred in the trunk and knee joint. This reflects the character of the discipline with four riders competing against each other over jumps and obstacles, creating more accidents due to collision and body contact than in other disciplines. The higher injury severity in this discipline is reflected by the highest number of days off due to injury, with 51.0 days. More evidence for the higher injury severity is the fact that almost half of all athletes in snowboardcross complain about impairments in their daily life.

Multiple studies were able to demonstrate the beneficial effect of protective gear in snowboarding. ${ }^{1,14,18,24-26}$ As $93 \%$ of all riders wore protective gear in this discipline, a reduction of injuries through protective gear does not seem to be a promising strategy and highlights the dangerousness of this discipline.

\section{Retired professional snowboarders}

The global injury-distribution in retired professional snowboarders was almost similar to the distribution in active professional snowboarders. The rate of wearing protective gear was significantly lower in this group compared to active professionals. This may be the result of the obligation to wear protective gear in competitive snowboarding in the late 1990s. A major difference related to the group of active professionals was seen in the number of days off. Here, the group of retired professional snowboarders stated a mean of 79.1 days off. It is tempting to speculate that a possible reason for this may be the increasing professionalization of the snowboarding sport with a more secure coverage of the contest areas and safer building of race tracks, better medical care in the national teams, and increasing pressure to regain physical health and continue to compete in contests. Interestingly, a great number of retired 
professionals stated impairment in their daily activities and a significantly high number of athletes were unable to regain the same snowboarding skills as that of the preinjury state. Twenty six percent even had to abandon their career due to injury reasons, and two athletes are not able to snowboard anymore. These results are very important because to our knowledge no data exist on the long-term risks and outcomes of professional snowboarding. With the active professional career being over in 8 years on average in our collective of retired professionals, our results can be regarded as meaningful as far as the aspect of a possible evolution of secondary health damages (osteoarthritis or persistent joint instability) on the base of injuries. Overall, the significant results related to retired professionals with a higher number of days off, a smaller rate of wearing protective gear, and a significantly higher number of athletes with inability to snowboard on the same level as that before injury, display the evolution and increasing professionalization of snowboarding during the last decades that have made it a high-performance sport similar to other well-established winter sports.

\section{Limitations of the study}

Besides the relatively small number of athletes and injuries, a possible bias is that athletes may not have stated all sustained injuries, remembering only the more severe ones and therefore over-interpreting the global injury severity. Professional snowboarders may also underestimate their actual impairment, as their career is not over yet. Due to the retrospective character of the study, the recall bias should be mentioned; additionally, the different study populations may have affected the completion of the questionnaire.

Furthermore, the prescreening of the collective of recreational snowboarders through medical centers and general practitioners near the ski resorts may be the reason for the higher injury severity observed in our cohort. Therefore, our recreational collective should not be seen as the "true" collective of all snowboard injuries, but as a collective that needs further treatment by specialists due to the severity of the injury or a necessary surgical treatment.

\section{Conclusion}

For the first time, injury distribution was compared among recreational, active, and retired professional snowboarders. Besides the confirmation of discipline-specific cumulation of various injuries and a smaller injury severity in the speed disciplines, new surprising aspects such as the inconsistent use of protective gear were found. The analysis of data pertaining to retired professionals revealed a significantly higher number of former athletes with inability to perform on the same level as that before injury and a significantly higher number of days off compared to that for active professionals. This first description of long-term impairments after professional snowboarding highlights the importance of developing specific strategies to prevent daily life impairments after a professional sports career.

Knowledge about the usual injury patterns in snowboarding is important and is of special interest to everyone involved in the treatment of snowboard-related injuries and will help to sufficiently treat these patients.

\section{Disclosure}

The authors declare no conflict of interest in this work.

\section{References}

1. Ehrnthaller C, Gebhard F, Kusche H. [Typical injuries in snowboarding. Possible prevention strategies]. Unfallchirurg. 2014;117(1): 7-12.

2. Torjussen J, Bahr R. Injuries among elite snowboarders (FIS Snowboard World Cup). Br J Sports Med. 2006;40(3):230-234.

3. Kim S, Endres NK, Johnson RJ, Ettlinger CF, Shealy JE. Snowboarding injuries: trends over time and comparisons with alpine skiing injuries. Am J Sports Med. 2012;40(4):770-776.

4. Kollrack Y. Verletzungsmuster beim Snowboarden [Pattern of injuries in snowboarding]. ViaMedici Online. Stuttgart: Georg Thieme Verlag; 2011. German.

5. Chow TK, Corbett SW, Farstad DJ. Spectrum of injuries from snowboarding. J Trauma. 1996;41(2):321-325.

6. Davidson TM, Laliotis AT. Snowboarding injuries, a four-year study with comparison with alpine ski injuries. West J Med. 1996;164(3): 231-237.

7. Gammons M, Boynton M, Russell J, Wilkens K. On-mountain coverage of competitive skiing and snowboarding events. Curr Sports Med Rep. 2011;10(3):140-146.

8. Bladin C, Giddings P, Robinson M. Australian snowboard injury data base study. A four-year prospective study. Am J Sports Med. 1993;21(5): 701-704.

9. Gorschewsky O, Goertzen M, Zollinger H. Snowboardverletzungen [Snowboard injuries]. Dt Z Sportmed. 1994;453:109-122. German.

10. Ehrnthaller C, Gebhard F, Imhoff AB, Braun S. [Bony injuries of the shoulder girdle in snowboarding]. Unfallchirurg. 2014;117(1): $13-18$.

11. Shealy JE, Sundman PD. Snowboarding injuries on alpine slopes. In: Johnson RJ, Mote CD, Binet MH, editors. Skiing Trauma and Safety: Seventh International Symposium. Philadelphia: American Society for Testing and Materials; 1989:75-81.

12. Ogawa H, Sumi H, Sumi Y, Shimizu K. Skill level-specific differences in snowboarding-related injuries. Am J Sports Med. 2010;38(3): 532-537.

13. Steenstrup SE, Bere T, Florenes TW, Bahr R, Nordsletten L. Injury incidence in qualification runs versus final runs in FIS World Cup snowboard cross and ski cross. Br J Sports Med. 2011;45(16):1310-1314.

14. Machold W, Kwasny O, Gassler P, et al. Risk of injury through snowboarding. J Trauma. 2000;48(6):1109-1114.

15. Muller R, Brugger O, Mathys R, Stussi E. [Snowboarding accidents]. Sportverl Sportschaden. 2000;14(4):121-127.

16. Matsumoto K, Miyamoto K, Sumi H, Sumi Y, Shimizu K. Upper extremity injuries in snowboarding and skiing: a comparative study. Clin J Sport Med. 2002;12(6):354-359. 
17. Yamauchi K, Wakahara K, Fukuta M, et al. Characteristics of upper extremity injuries sustained by falling during snowboarding: a study of 1918 cases. Am J Sports Med. 2010;38(7):1468-1474.

18. Idzikowski JR, Janes PC, Abbott PJ. Upper extremity snowboarding injuries. Ten-year results from the Colorado snowboard injury survey. Am J Sports Med. 2000;28(6):825-832.

19. Oberthaler G, Primavesi C, Niederwieser B, Hertz H. [Snowboarding accidents 1991 to 1994 - an analysis]. Sportverl Sportschaden. 1995;9(4):118-122.

20. Yamakawa H, Murase S, Sakai H, et al. Spinal injuries in snowboarders: risk of jumping as an integral part of snowboarding. J Trauma. 2001; 50(6):1101-1105.

21. Torjussen J, Bahr R. Injuries among competitive snowboarders at the national elite level. Am J Sports Med. 2005;33(3):370-377.
22. Florenes TW, Nordsletten L, Heir S, Bahr R. Recording injuries among World Cup skiers and snowboarders: a methodological study. Scand J Med Sci Sports. 2011;21(2):196-205.

23. Kirkpatrick DP, Hunter RE, Janes PC, Mastrangelo J, Nicholas RA. The snowboarder's foot and ankle. Am J Sports Med. 1998;26(2) 271-277.

24. Nakaguchi H, Fujimaki T, Ueki K, Takahashi M, Yoshida H, Kirino T. Snowboard head injury: prospective study in Chino, Nagano, for two seasons from 1995 to 1997. J Trauma. 1999;46(6):1066-1069.

25. Pino EC, Colville MR. Snowboard injuries. Am J Sports Med. 1989; 17(6):778-781.

26. Ronning R, Ronning I, Gerner T, Engebretsen L. The efficacy of wrist protectors in preventing snowboarding injuries. Am J Sports Med. 2001; 29(5):581-585.

\section{Publish your work in this journal}

Open Access Journal of Sports Medicine is an international, peer-reviewed, open access journal publishing original research, reports, reviews and commentaries on all areas of sports medicine. The manuscript management system is completely online and includes a very quick and fair peer-review system.
Visit http://www.dovepress.com/testimonials.php to read real quotes from published authors. 